Supporting Information to:

\title{
Compressive Broad-Band Sum Frequency Generation Microscopy to Study Functionalized Surfaces
}

\author{
Desheng Zheng, Liyang Lu, Yun Li, Kevin F. Kelly*, and Steven Baldelli* \\ Department of Chemistry, University of Houston, Houston, TX 77204-5003 \\ Department of Electrical and Computer Engineering, Rice University, Houston, TX 77005
}

1. Instrumentation

The home build CS-SFG microscopy was designed in the Oblique configuration to adapt to the conventional SFG spectroscopy measurement. Figure 1 depicts the schematic layout of the structure illuminated CS-SFG microscope. Briefly, the picosecond visible pulse beam from SHBC expanded and collimated with beam perpendicularly incident on the grating G1, the first order diffraction beam has been reflected to the digital micro-mirror device (DMD, Texas Instrument Discovery 4100 ) with incident angle at $24^{\circ}$. After the DMD pattern reflection, the spatially modulated visible beam was reduced by tube lens (TL) and objective lens (Obj), then perpendicularly incident on the grating G2. The first order diffraction beam after G2 is illuminated on the sample after passing the 1:1 relay lens RL1. The femtosecond IR beam from TOPAS has incidents on the sample from contour direction. The visible beam and IR beam have overlapped temporarily and spatially on the sample at an incident angle (relative to the surface normal) of $60^{\circ}$. The generated SF signal has passed the 1:1 Relay lens 2 and is reflected to the spectrograph by mirror 5, 6, 7, and 8. Relay Lens L2 with larger NA has been used to adapt the variation of the signal direction as the wavelength tuning of IR beam. L3 focus the SFG signal to the slit of the spectrograph. After the Spectrograph, the SFG signal has been record by liquid nitrogen cooled CCD (PyLoN 100B/LN, Princeton Instrument).

In current microscope design, the compressive sampling has been achieved through modulating the Vis beam profiles as permuted Walsh-Hadamard patterns via digital micromirror device. The spatial patterned SFG signal from the sample surface has been guided to the spectrograph. Then the broad band SFG spectrum from each consecutive permuted WalshHadamard patterns has been recorded synchronized. With enough iterations of DMD patterns to obtain a tolerable image quality, the hyperspectral image cube could be reconstructed through the underdetermined minimization algorithms. The gain in image speed thus results from the ability to capture imaging information while limiting the total number of acquisitions by exploiting sparsity in the image, which could be well judged from the image quality at different compression ratios. 
As in Figure 1S, the grating G1 is used to generate the difference of optical distance d0, which will compensate variations of optical distance $d 1$, which is induced by the tilted incident on the DMD surface and the contour incident to the sample relative to the IR beam $\mathrm{d} 2$, as illustrated in Figure 1. This compensation is important for increased view field of this microscope, by max to temporal and spatial overlap with the femtosecond IR pulse laser at the sample surface. Grating G2 has been used to correct the rectangle distortion from the oblique excitation on the sample. To synchronize the DMD pattern with the CCD recording, the LN-CCD has been external triggered by the DMD TTL sync-out. The current CS-SFG microscopy has the spectral resolution about $8 \mathrm{~cm}^{-1}$, and lateral resolution about 2 um with DMD finest binning.
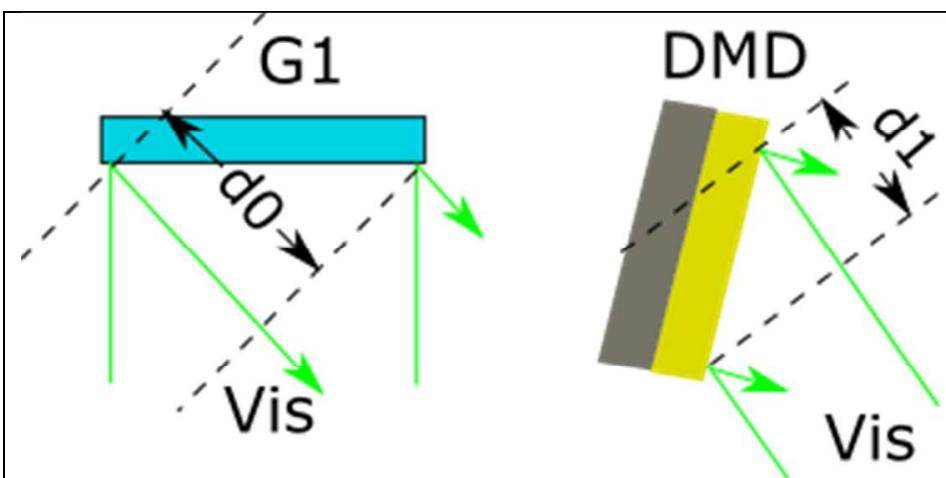

\section{Sample}

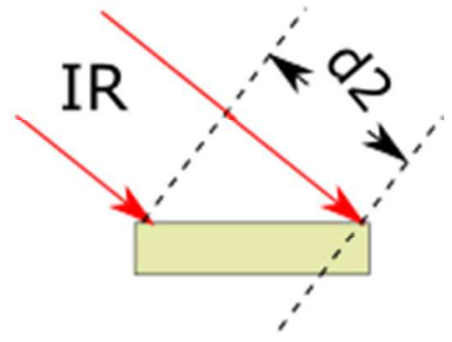

Figure S1 Schematic diagram of timing compensation for the oblique counter propagation configuration. G1 the Plane Ruled Reflectance Gratings; d0 is the optical distance for vis beam; $\mathrm{d} 1$ is the optical distance generated from the Vis beam from DMD; $\mathrm{d} 2$ is the optical distance for oblique illumination IR beam. For better view field, $\mathrm{d} 0$ should compensate the $\mathrm{d} 1$ and $\mathrm{d} 2$, $\mathrm{d} 0=\mathrm{d} 1+\mathrm{d} 2$.

\section{Normalized mean square error}

The quality of the image as a function of percentage of DMD patterns was estimated using the normalized mean square error (NMSE). The normalized mean square error is defined as

$$
N M S E=\frac{\sum_{1}^{n}\left(A_{i}-S T_{i}\right)^{2}}{\sum_{1}^{n}\left(S T_{i}\right)^{2}}
$$

Here, $A$ is a one dimensional vector with n elements reshaped from the analyzed image matrix, and, ST is a one dimensional vector with $\mathrm{n}$ elements reshaped from the standard image matrix. Since it is indeed difficult to strictly define and analyze noises because we do not have the noiseless ground truth, the standard image was defined as the image acquired and reconstructed by 3DTV algorithm, using 15564 DMD testing patterns (95\% of 16384) at a resolution of $128 \times$ 128pixels. The NMSE of the images recovered by different algorithms at $95 \%, 50 \%, 25 \%$, 
$12.5 \%, 6.25 \%$, and $3.125 \%$, of the total patterns were calculated respectively. As shown in figure $5 \mathrm{~b}$, the visual image quality improved significantly as the number of patterns increased.

3. Comparison of the imaging speed

The imaging speeds of three SFG microspores in our lab have been compared. These three SFG microspores are narrow band CS-SFG microscope, narrow band SFG microscope and broad band CS-SFG microscope, respectively. The pump beam of narrow band CS-SFG microscope and narrow band SFG microscope is picosecond pulse at $1064 \mathrm{~nm}$. The pump beam of broad band CS-SFG microscope is picosecond pulse at $514 \mathrm{~nm}$. Table S1 has shown the basic imaging parameters of these three SFG microscopes, which could generate images with reasonable quality. Comparing to narrow band CS-SFG microscope, the imaging speed of broad band CSSFG microscope is about 200 times increasing, beside the advantages of low pulse energy of pump beam. Comparing to narrow band SFG microscope, broad band CS-SFG microscope has the competitive imaging speed, beside the advantages of low pulse energy of pump beam. Pulse energy of the pump bean is an important factor, when considering the stability of samples especially on metal surfaces. Here only the rough comparison is provided. Quantitatively comparisons in details of this three microscopes is still necessary in the future.

\begin{tabular}{|c|c|c|c|}
\hline & $\begin{array}{l}\text { Narrow band } \\
\text { CS-SFG } \\
\text { microscope** }\end{array}$ & $\begin{array}{l}\text { Narrow band } \\
\text { SFG microscope }\end{array}$ & $\begin{array}{l}\text { Broad band CS-SFG } \\
\text { microscope } * *\end{array}$ \\
\hline pixels & $64 \times 64$ & $64 \times 64$ & $64 \times 64$ \\
\hline Pulse repetition & $20 \mathrm{~Hz}$ & $20 \mathrm{~Hz}$ & $5 \mathrm{kHz}$ \\
\hline $\begin{array}{l}\text { Pump beam } \\
\text { pulse energy }\end{array}$ & $1000 \mathrm{uj}$ & $1000 \mathrm{uj}$ & $0.4 \mathrm{uj}$ \\
\hline \#shots/pattern & 150 & 1000 & 2500 \\
\hline Spectral resolution & $6 \mathrm{~cm}^{-1}$ & $6 \mathrm{~cm}^{-1}$ & $8 \mathrm{~cm}^{-1}$ \\
\hline Spectral range & & & $120 \mathrm{~cm}^{-1}$ \\
\hline $\begin{array}{l}\text { Time data } \\
\text { acquisition }\end{array}$ & $30720 \mathrm{~s}$ & $50 \mathrm{~s}$ & $2048 s$ \\
\hline $\begin{array}{l}\text { Image number per } \\
\text { measurement }\end{array}$ & 1 frame & 1 frame & 15 frames \\
\hline Imaging speed & $\begin{array}{c}0.12 \\
\text { frame/hour }\end{array}$ & 72 frames/hour & 26 frames/hour \\
\hline
\end{tabular}

Table S1. Imaging parameters for narrow band CS-SFG microscope, narrow band SFG microscope and Broad band CS-SFG microscope. **All the speed compared at $\mathbf{1 0 0 \%}$ percentage of patterns without compression.

4. Comparison between raster scanning and the compressive sampling 
Gold pattern on silicon wafer has been used to demonstrate the imaging qualities of raster scanning and the compressive sampling. In the raster scanning measurement, each DMD pattern only has one pixel has been used to reflect the visible beam to the sample. The one pixel is a binning of $6 \times 6$ micromirrors. During the measurements of raster scanning and compressive sampling, power of Vis beam was set at $1.5 \mathrm{mw}$, and the power of IR was set at $50 \mathrm{mw}$. Both beams incident on the sample with area about $0.25 \mathrm{~mm}^{2}$. The exposure time of CCD was set at 1 second/frame. Figure S2 (A) has shown the Typical SFG intensity curves from Raster scanning and compressive sensing at each pattern. Figure S2 (B) has shown the Image reconstructed based on Raster scanning at $2860 \mathrm{~cm}^{-1}$, and Figure S2 (C) has shown Image at $2860 \mathrm{~cm}^{-1}$ reconstructed based on compressive sensing with $100 \%$ of 4096 frames. Both spectra and images have demonstrated that signal noise ratio of compressive sampling are dramatically higher than that of raster scanning, since there are 2048 pixels are reflecting the visible beam to the sample in compressive sampling rather than only 1 pixel reflecting the visible beam to the sample.

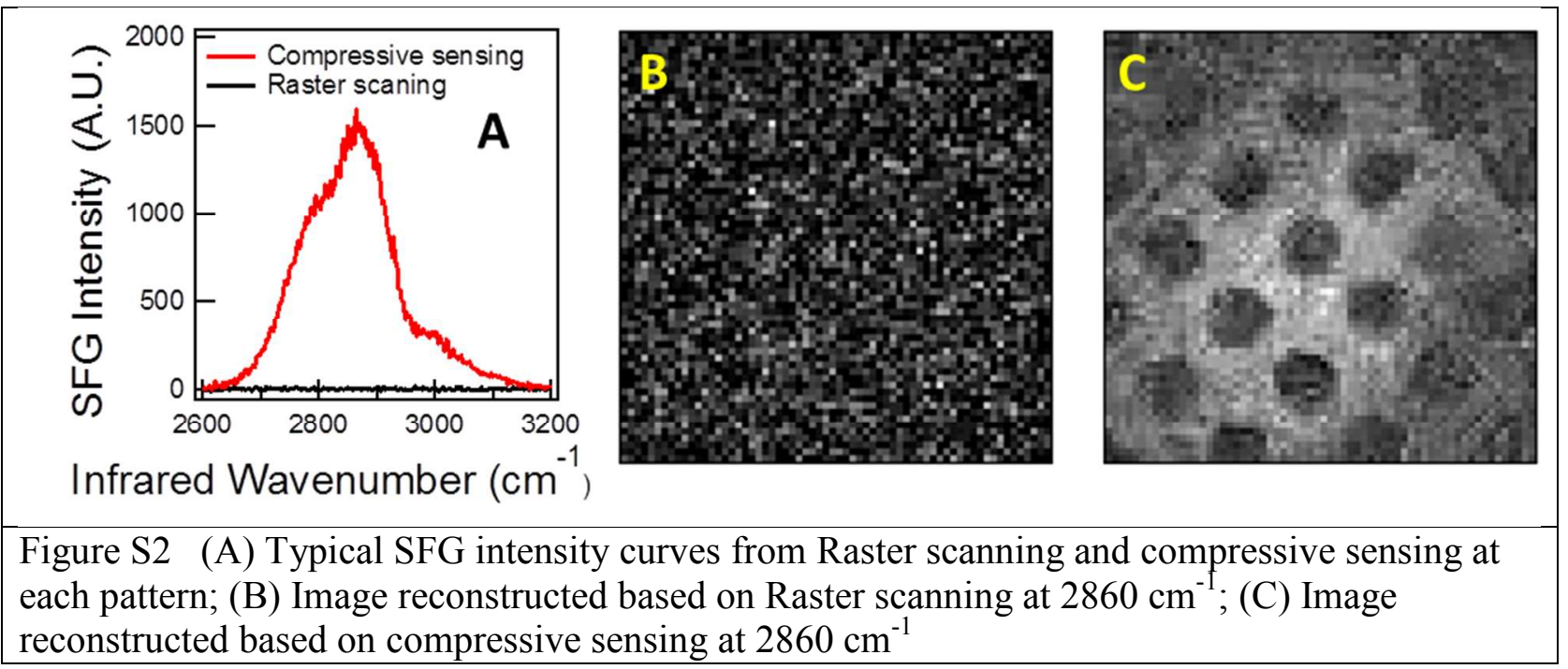

5. Peak Signal-to-Noise Ratio

Peak signal-to-Noise Ratio (PSNR) is another measure generally used as of the image quality in compressive sensing. Although a higher PSNR generally indicates the higher quality of reconstruction, the validity of this measure is only conclusively suitable to compare results from the same algorithm and content. The definition of PSNR is as

$$
P S N R=10 \log _{10} \frac{[\operatorname{Max}(S T)-\operatorname{Min}(S T)]^{2}}{\left[\sum_{1}^{n}\left(A_{i}-S T_{i}\right)^{2}\right] / n}
$$


Here, $A$ is a one dimensional vector with $\mathrm{n}$ elements reshaped from the analyzed image matrix, and, $\mathrm{ST}$ is a one dimensional vector with $\mathrm{n}$ elements reshaped from the standard image matrix. In our analysis, the standard image was still defined as the image reconstructed by 3DTV algorithm with $95 \%$ measurement percentage, since it is indeed difficult to strictly define and analyze noises because we do not have the noiseless ground truth. The PSNR of the images recovered by 3DTV algorithm at $50 \%, 25 \%, 12.5 \%, 6.25 \%$, and $3.125 \%$, of the total patterns were calculated respectively, as shown in Figure S3. The visual image quality improved significantly as the number of patterns increased.

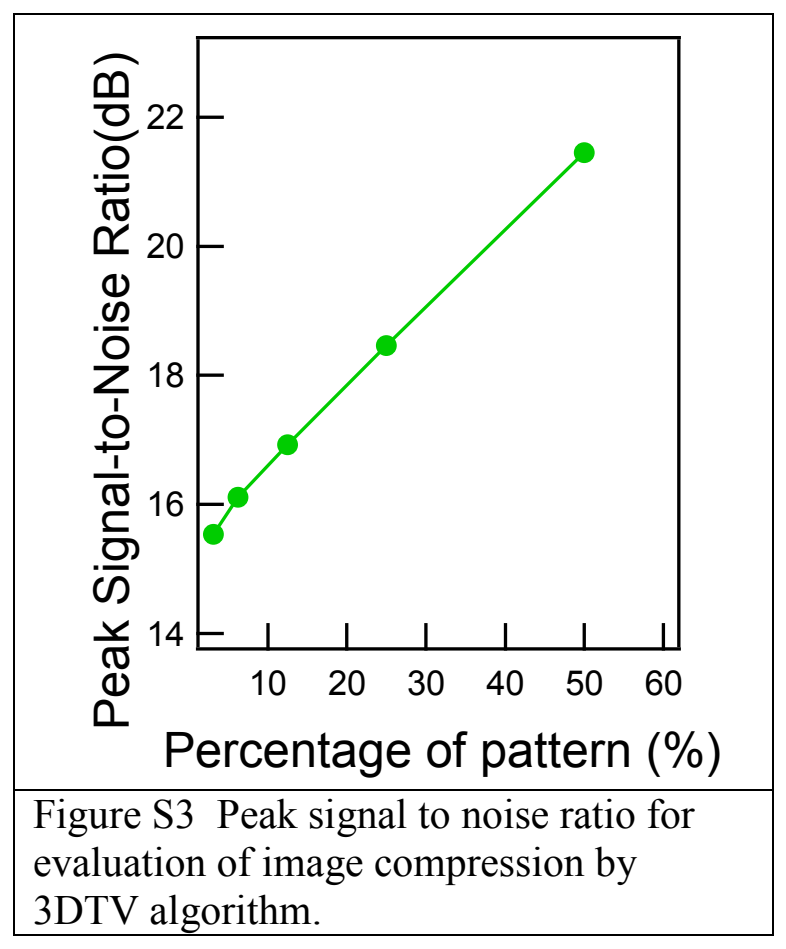

